\title{
Frailty, fitness and late-life mortality in relation to chronological and biological age Arnold B Mitnitski ${ }^{1}$, Janice E Graham², Alexander J Mogilner ${ }^{3}$ and Kenneth Rockwood*4
}

Address: ${ }^{1}$ École Polytechnique, Montreal QB, Canada, ${ }^{2}$ Department of Anthropology and Sociology, University of British Columbia, Vancouver BC, Canada, ${ }^{3}$ Montreal, QB, Canada and ${ }^{4}$ Division of Geriatric Medicine, Dalhousie University, Halifax, NS, Canada

E-mail: Arnold B Mitnitski - arnold@grbb.polymtl.ca; Janice J Graham - igraham@interchange.ubc.ca; Alexander E Mogilner - alex.mog@rocketmail.com; Kenneth Rockwood* - rockwood@is.dal.ca

${ }^{*}$ Corresponding author

Published: 27 February 2002

BMC Geriatrics 2002, 2:I
Received: |4 December 200|

Accepted: 27 February 2002

This article is available from: http://www.biomedcentral.com//47|-23/8/2/I

(c) 2002 Mitnitski et al; licensee BioMed Central Ltd. Verbatim copying and redistribution of this article are permitted in any medium for any purpose, provided this notice is preserved along with the article's original URL.

\begin{abstract}
Background: People age at remarkably different rates, but how to estimate trajectories of senescence is controversial.

Methods: In a secondary analysis of a representative cohort of Canadians aged 65 and over $(\mathrm{n}=$ 29/4) we estimated a frailty index based on the proportion of 20 deficits observed in a structured clinical examination. The construct validity of the index was examined through its relationship to chronological age (CA). The criterion validity was examined in its ability to predict mortality, and in relation to other predictions about aging. From the frailty index, relative (to CA) fitness and frailty were estimated, as was an individual's biological age.

Results: The average value of the frailty index increased with age in a log-linear relationship $(r=$ $0.9 \mathrm{I} ; \mathrm{p}<0.00 \mathrm{I}$ ). In a Cox regression analysis, biological age was significantly more highly associated with death than chronological age. The average increase in the frailty index (i.e. the average accumulation of deficits) amongst those with no cognitive impairment was 3 per cent per year.

Conclusions: The frailty index is a sensitive predictor of survival. As the index includes items not traditionally related to adverse health outcomes, the finding is compatible with a view of frailty as the failure to integrate the complex responses required to maintain function.
\end{abstract}

\section{Background}

Although the prevalence of both illness and functional impairment rise with age, individuals with same chronological age vary widely in health and function [1]. How best to summarize this variability in impairments is not clear. While functional disabilities tend to follow a hierarchical pattern, [2-4] summarizing disabilities without reference to the associated illnesses omits important information. Broader concepts, with a more rigorous the- oretical base, such as frailty [1] and allostatic load [5], have been proposed as a better means of assessing the heterogeneity of health status amongst elderly people, but none has yet proved entirely satisfactory [6-9].

We recently proposed that the concept of functional age can be derived from a representative database which includes information on a range of variables [10]. Earlier, we identified a constellation of signs and symptoms from 
this database which show synergy (close inter-relatedness in complex patterns) in health and loss of synergy in neurodegenerative disease [11]. Recalling that both advanced age and the frail state are associated with loss of complexity and resiliency and, ultimately, with death $[7,12-15]$ we now extend these observations to propose that, as a first approximation, an individual's health status can be quantified as the proportion of ageing associated deficits which they have incurred. Further, we suggest that both fitness and frailty can be estimated by comparing the number of symptoms and signs (jointly referred to herein as deficits) which are present in an individual with the mean number of deficits present in others of the same chronological age.

\section{Materials and methods Sample}

These data come from the inception cohort of the Canadian Study of Health and Aging (CSHA) [16], a representative survey of people aged 65 and over. The chief goals of the CSHA were to identify the prevalence, incidence outcomes of and risks for dementia. In the first phase (CSHA1) data collection took place between February 1991 and May 1992. In Figure 1, the sample population is presented as a flow chart. Initially, 10,267 people were interviewed - 9,008 in the community, and the remainder in longterm care institutions. The response rate in the community was $72 \%$ and was $82 \%$ for those in long-term care. Participants in the clinical component were selected from a random sample of elderly Canadians, based on their score on the Modified Mini-Mental Status Examination (3 MS) [17]. Those who screened positive, and a sample who screened negative were invited to a clinical examination designed to detect cognitive impairment and diagnose its cause $(\mathrm{n}=2914)$. Demographic and diagnostic details have appeared elsewhere [16,18-20]. Briefly, of the 2914 who came for a clinical examination $64.4 \%$ were women. Their mean age at baseline was 82.0 years; SD 7.43, range 65-106. Dementia, and its subtypes, were diagnosed and staged by standard protocols, $[16,19,20]$ based on the examination and an informant interview, in 1132 people. Of the remainder, 861 were classified as having Cognitive Impairment No Dementia (CIND) [19] and 921 with no cognitive impairment. All 1338 surviving clinical participants received a follow-up examination approximately five years after the baseline assessment [20]. Date of death was recorded for 1465 of the 1521 subjects who did not survive. The median time to death was 33 months.

\section{Selection of deficits}

To select the signs and symptoms for analysis, we have had to compromise between the desire to use as many items as possible from the database (more than 400 items were available) and the need to minimize noise due to measurement error, including missing data. Therefore, at this early stage of our investigations, we have restricted ourselves to deficits that represent a variety of functions, and at the same time, occurred with reasonable frequency (on average, in $26.7 \%$ of subjects) had minimum (on average $12 \%$ ) missing data, and maximum variability across the database. Our earliest studies demonstrated that taking into account from 19 to 26 signs and symptoms (depending on the specific applications) we were able to address different aspects of cognitive aging from diagnostics $[21,22]$ to functional decline [23]. The most important finding of the initial studies was the demonstration that the deficits are not independent. In other words, the variables are closely interrelated and, in a sense, any deficit contains some information about many of the others [11]. This implies that even a restricted set of deficits, which show the above properties can represent a wide variety of impairments. In a prior analysis, we identified a set of 20 symptoms, signs, impairments and disabilities (referred to collectively as deficits) that represent loss of functional activities, sensory impairment, and general medical, health and behavioural problems $[10,11]$. The variables represent deficits, which are more common with age and include informant-based (e.g. data on function from CAMDEX H) [16] observer-assessed (e.g. clinical reports of hypertension, diabetes) and test (e.g. $3 \mathrm{MS}$ ) data.

The list of deficits is presented below. (The numbers in parentheses refers to their position in Figure 6 and correspond to those used in [27]). Vision loss (22), hearing loss (23), impaired mobility (3), vascular problem (36), gait abnormality, impaired vibration sense (56), difficulty toileting (11), difficulty cooking (7), difficulty bathing (10), difficulty going out (6), difficulty grooming (9), skin problems (40), resting tremor (47), changes in sleep (2), difficulty dressing (8), urinary complaints (29), gastro-intestinal problem (28), diabetes (31), hypertension (24), limb tone abnormality (46).

\section{Analysis}

We considered binary deficits (represented by either 1 or 0 ), depending on their presence or absence in a given individual. For simplicity, we considered the proportion of the deficits in the $i$-th individual $\left(0<q_{i}<1\right)$ as a state variable, measuring an individual degree of impairment and frailty. We analyzed the proportion $q(t)$ averaged across all subjects at age $t$, for those with no cognitive impairment and checked the hypothesis of the linear increase of $\ln (q)$ with age, as a test of the construct validity of our model. Regression techniques were applied to estimate the parameters of the linear model. Cox proportional hazards regression with covariates and Gompertz's model were used in analysis of the survival data to test the criterion validity of the index in relation to death. The significance level was set at $p<0.05$. 


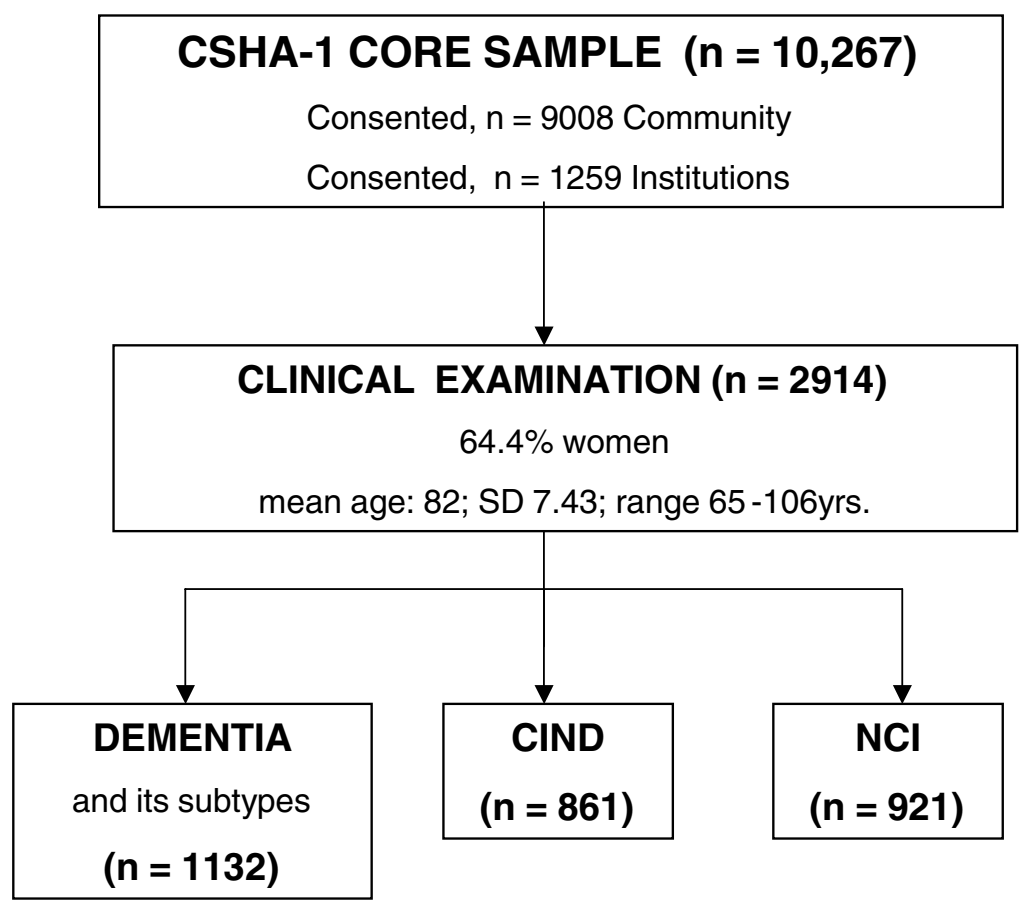

Figure I

Population sample flow chart.

\section{Results}

The impairment index, $q$ ranged from 0 to 1 . At any given age, the impairment index is distributed about the mean value for individuals of that age. Figure 2 illustrates this for subjects aged 77 years old who did not demonstrate cognitive impairment. An individual's health status, $f_{i}$ may be defined as a ratio of that person's impairment index to the mean index value, averaged across individuals without cognitive impairment, but of the same chronological age: $f_{i}=q_{i} / m ; f>1$ if the individual is frail and $f<$ 1 if the individual is fit. The logarithm of the ratio $\ln (f)$ may also be considered as an appropriate index of relative frailty/fitness; a positive value of the logarithm indicates frailty, whereas a negative value indicates fitness. (When relative fitness/frailty is to be measured as a dichotomous variable, the mean case is taken as fit; i.e. $f<1$.) Figure 3 presents the proportion of fit and frail people who survive, averaged by 5 -years intervals. As expected, this decreases with age for both groups, but note that a higher proportion of fit individuals than frail survive in each age group.

Figure 4 presents the log of the mean value of the frailty index. Consistent with our hypothesis, the frailty index value increases with age. Because age and the frailty index are well correlated $(r=0.91, p<0.001)$ we can use this information to estimate relative fitness and frailty, with rea- sonable precision, across the age spectrum. The regression line corresponds to the equation:

$\ln (q)=-4.23+0.03 t$

For example, according to equation (1), after elementary calculations, 67, 77 and 87 year old individuals expect to have the following proportions of deficits, $m=0.11,0.15$ and 0.2 , respectively. Now consider a 67 individual with 3 deficits from the list of 20 . The proportion of deficits $q$ $=0.15$, and therefore such individual may be considered as frail since $q>m=0.11$. However, a 87 years old individual with the same $q$ would be considered as fit, since $q$ $<m=0.2$. In other words, the frailty index represents relative fitness and frailty, so that a given degree of fitness at age 87 may represent frailty at age 67 .

A separate, but related way of quantifying relative frailty and fitness is to use equation (1) as a calibration tool, which again can be done because it holds with a satisfactory degree of certainty ( $r=0.91$ corresponding to $83 \%$ of explained variance). By inverting equation (1) and inputting the proportion of deficits, $q$ we arrive at a value of age $t$ as an output. Calculated in this way, the biological age of an individual can be understood as the age at which the average individual has $q$ deficits. Similarly to equation (1) a precise estimate of relative fitness and frailty can be de- 


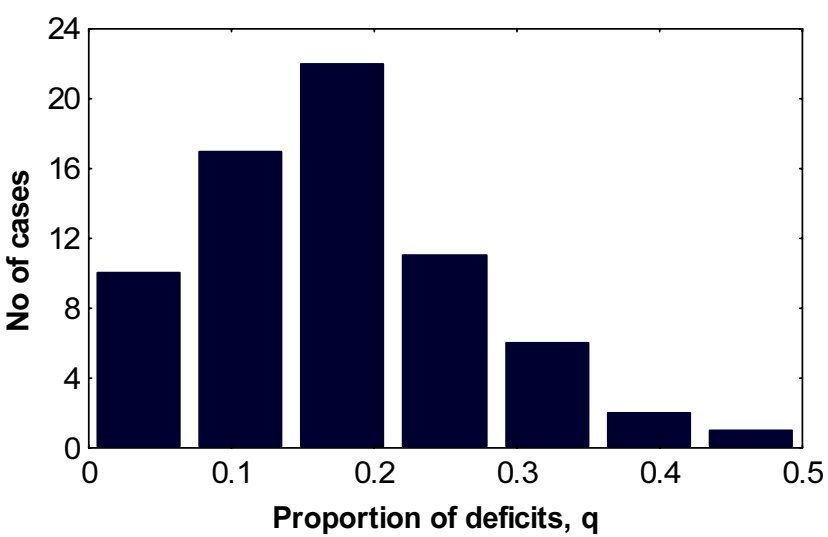

Figure 2

The distribution of the frailty index at chronological age 77 suggests varying levels of fitness and frailty, even in those with no cognitive impairment.

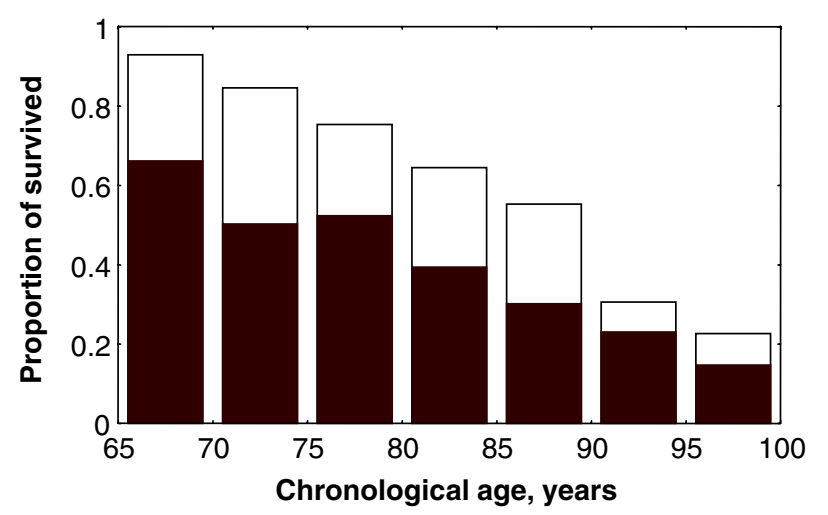

Figure 3

The proportion of survivals for frail (solid rectangles) and fit individuals (total rectangles) decreases with the chronological age. However, frail individuals show lower survival at all age groups than do fit individuals.

rived: if the calculated age is less than that individual's chronological age, he/she is considered fit, if it is greater than the average age at which people have such deficits, otherwise he/she considered frail. Such a definition of frailty therefore depends on two inputs: chronological age and the proportion of deficits seen at any given age. The difference between the individual's chronological age and the expected age given the proportion of deficits can be used to assess a given individual's relative frailty or fitness. Recalling the log-linear relationship between the impairment index and chronological age (Figure 4), we suggest that this value can be used as a first approximation in the

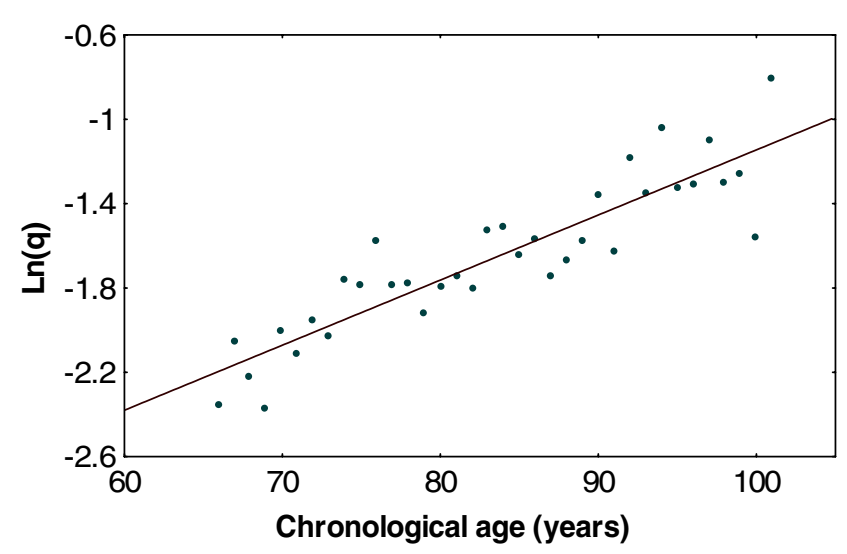

Figure 4

Mean proportion of deficits at any given age for subjects with no cognitive impairment. Solid circles represent the proportion of the deficits averaged across all subjects at age $C A$ : In (q) $=-4.23+0.03$ CA. Correlation coefficient, $r=0.91, p<$ 0.0001

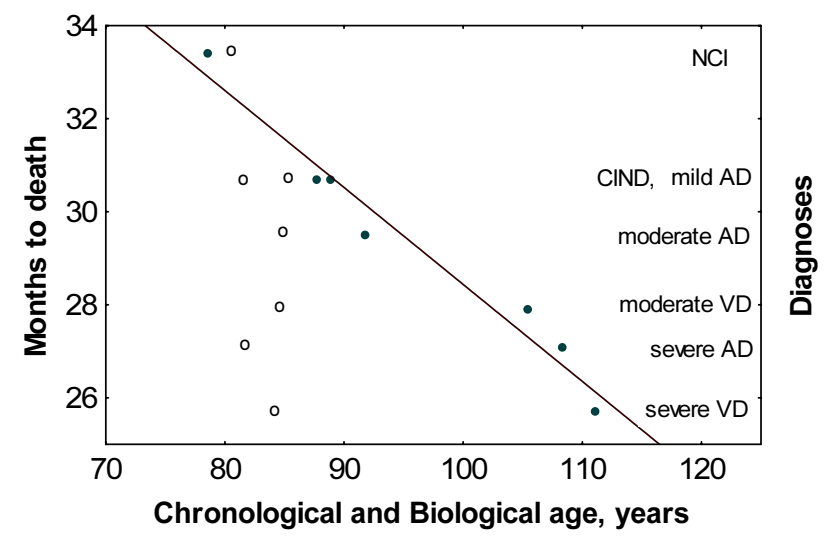

Figure 5

Time to death by cognitive diagnostic groups as a function of chronological and biological age. Solid circles correspond to average values of time to death (across all subjects of the particular diagnostic group) with respect to averaged $B A$ distributed along a straight line $(r=-0.98, p<0.001)$. Empty circles, (o) correspond to time to death with respect to $C A$ and did not show such a pattern. The following abbreviations are used for the diagnostic groups: $\mathrm{NCl}$ (No Cognitive Impairment), CIND (Cognitive Impairment No Dementia), AD (Alzheimer's disease), VD (vascular dementia).

calculation of personal biological age (PBA) by applying an inverse regression, (regression of age on $\ln (q)$ ), so that:

$P B A=126.65+26.09 \ln (q)$ 
An individual's $P B A$ is defined as the value at which those deficits are present, on average, in the successfully ageing group. Chronological (CA) and biological age coincide when an individual's number of deficits corresponds to the age given by the equation above. Otherwise, an individual may be biologically younger (more fit) or older (more frail) than their chronological age, and the degree of relative fitness or frailty can be estimated as the difference between $P B A$ and $C A$. For example, a person with 4 deficits from $20(q=0.20)$, according to formula (2) has an estimated PBA of 83 years. Comparing this value with his/her chronological age one may consider him/her fit or frail depending on the difference between PBA and chronological age.

Figure 5 presents the mean months to death, plotted against mean chronological and biological age, for the main CSHA cognitive diagnostic groups. As hypothesised, biological age predicts death in a dose-response relationship. While chronological age has only a weak relationship to time to death, given diagnosis, biological age is strongly inversely correlated with the time to death $(r=$ $0.98, p<0.01)$.

PBA and CA were compared in relation to survival using Cox regression, with each as covariates (Table 1). Note that while the regression coefficients of CA and PBA are the same, the standard errors are quite different, so that PBA shows a much stronger relationship with mortality. Figure 6 serves as a further test of the ability of PBA and CA to predict mortality. A least squares estimates of the survival functions using a Gompertz model was fitted to the survival data for two groups (younger than 80 years and older than 80 years) for each of CA (panel A) and PBA (panel B). (The same relationships hold when other ages are used to dichotomize the sample.) The survival functions clearly show greater differences with PBA compared with CA, indicating a more refined ability to discriminate adverse outcomes with the former.

\section{Discussion}

We have extended an earlier conceptualization of functional age [10] to present a method of estimating personal biological age, and from that, to estimate relative fitness and frailty. In particular, we claim that the proportion of deficits accumulated by an individual at a given chronological age allows an operational definition of relative fitness and frailty. To validate this definition of relative fitness and frailty we compared survival both as function of PBA in the cohort, and by varying levels of fitness and frailty within cognitive diagnostic groups.

Calibration equations (1) and (2) comprise our knowledge about the accumulation of deficits in older adults, and thus can be considered as an example of knowledge

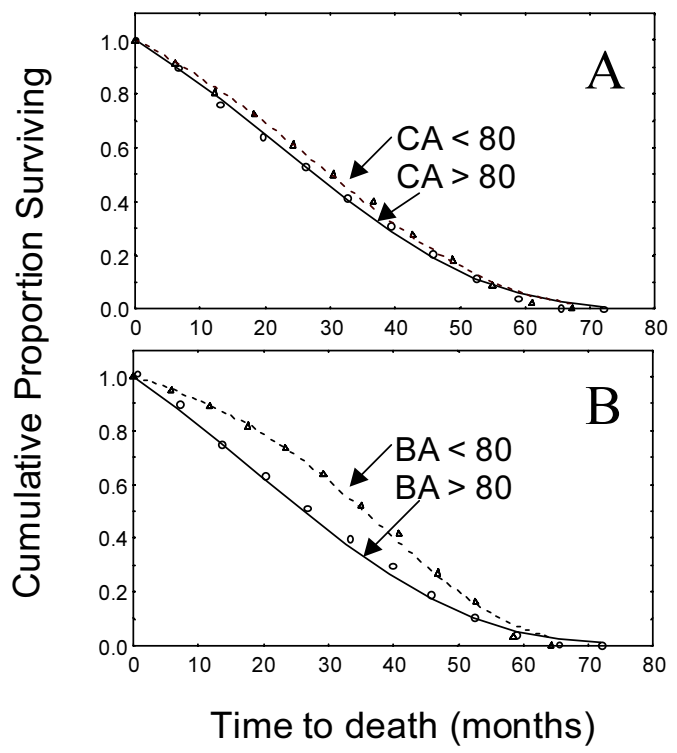

\section{Figure 6}

Cumulative proportion of surviving as a function of time to death for two groups with chronological age $(A)$ and biologi$\mathrm{cal}$ age $(\mathrm{B})$ less and greater than 80 years. Circles correspond to experimental data for individuals below 80 years old and triangles for individuals above 80 years. Curves correspond to the least square Gompertz's functions (solid lines fit data for the individuals below 80 years and dashed lines for those who was older than 80 years).

discovery in databases (KDD). KDD is a set of techniques being developed through a number of disciplines to take advantage of existing databases as means of discovering new knowledge. $[24,25]$ KDD, defined as the identification of meaningful and useful patterns in databases, includes both the discovery of previously unseen groupings within existing databases $[11,22]$ and predictive modelling, as also developed in this inquiry.

We are aware of important limitations in our study. First, in order to initially describe a simple model, we have limited our analysis to a set of 20 deficits. While these proved sufficient in earlier analyses, [21-23] alternate formulations of the impairment index may be more efficient. The more essential the deficits that are taken into account, the more precise the estimation of frailty that is obtained [26]. However, it is not yet clear which are the essential properties of signs, symptoms and functional deficits that need to be selected. This is an ongoing area of investigation, but it appears that the number of deficits, rather than their precise nature, might be the most important determinant $[26,27]$. We interpret this to mean that frailty might be interpreted as a loss of redundancy in a complex system. 
Table I: Parameters of Cox regression model for time to death as a function of CA and BA tag for table legends and titles

\begin{tabular}{cccccc}
\hline Variable & Beta & STD & t-value & Wald statist & p-value \\
\hline CA & 0.0081 & 0.0038 & 2.15 & 4.63 & 0.0313 \\
BA & 0.0081 & 0.0014 & 5.70 & 32.49 & $<0.000001$
\end{tabular}

In addition, we were limited in our databases to individuals aged 65 years and older, and drew from the screened clinical sample, so that we cannot make a claim about the representativeness of the data. The incorporation of data on middle-aged and representative samples should allow more general claims about $P B A$ to be examined. Third, to simplify the calculations we have suggested, as a first approximation, that a state variable can be estimated as the proportion of deficits. This may seem naive, as if has the effect of equalizing all the deficits. Evidently, at an individual level, not all deficits are equally important: heart problems or diabetes likely may cause death sooner than for example, difficulties in getting dressed or skin problems per se. The finding that the proportion of deficits in a given individual can include seemingly arbitrary or even trivial ones requires further investigation. For now, we understand this finding to mean that accumulating several deficits results overall in impaired adaptive ability. This is likely to be the case if the signs are redundant, i.e. if a given deficit represents a set of others, and if the items of the index are related. The latter appears to be the case, as illustrated in Figure 7, which shows that the deficits are not independent. The nodes of the graph correspond to the deficits (numbered in the Materials and methods) and the edges represent statistically significant relationships between the deficits (defined as the difference between the unconditional probability of the occurrence of deficit $X$ and conditional probability of deficit $\mathrm{X}$ given deficit $\mathrm{Y}$ ) [11]. This is not surprising when we consider that synergetic relationships are typical for age-associated deficits. In other words, roughly speaking, everything is dependent on everything else in complex organisms so that changes in one subsystem affects many others. For example, vision impairment may be caused by the numerous reasons. Since vision loss, by itself, is not readily regarded as a lifethreatening factor, it may indicate a more serious problem (e.g. diabetes, stroke). The more deficits that are used in deriving the frailty index, the greater the chance that such secondary signs are linked to serious illnesses. As argued elsewhere $[1,26,28]$ this is a central aspect of many characterizations of frailty. Moreover, whether this holds for any combination of deficits (and not just age-associated ones) additionally requires further study, although we recognize that such summarization does not allow the influence of individual disease states to be tested. [22,29]

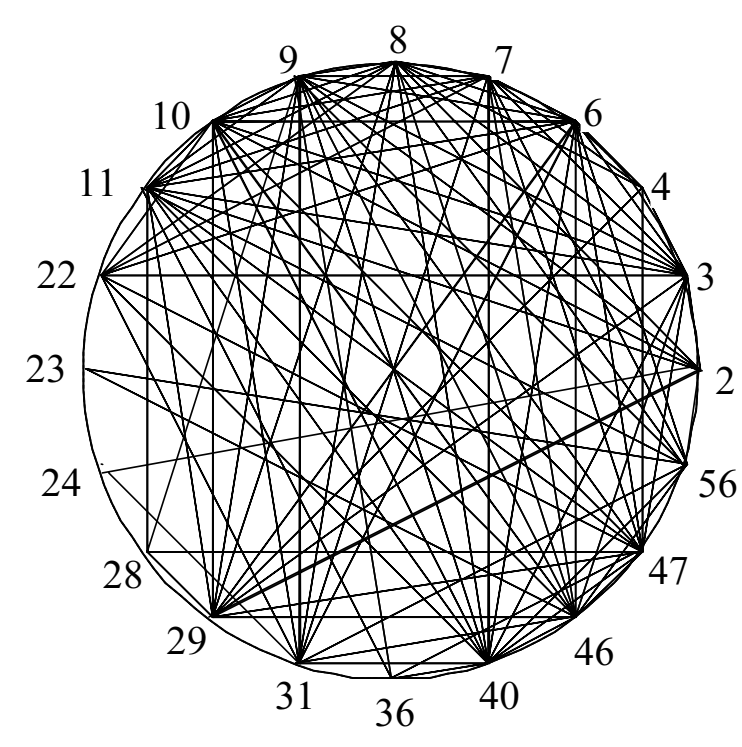

\section{Figure 7}

Inter signs synergy graph. Nodes indicate the deficits (codes correspond to the deficits from Methods and [29]) and edges indicate the statistically significant relationships between deficits, i.e. when the conditional probability of one deficit, given another is statistically different ( $p<0.05$; $t$-test) from the unconditional probability of the first deficit.

We considered data together for men and women. The differences in mortality of men and women are well known and we recognise that in the next approximation they have to be treated separately. However, here we were limited by the power of data representing, in average, 100 individuals at each age and division the sample by sex would make statistics worse. We intend to address the issue of gender differences in accumulation of deficits in the other paper when dealing with the representative large sample of data.

Have we offered any special insight beyond the commonsense observation that as people age they are more likely to become ill, or that ill people are more likely to die? We believe that we have. In the first instance, we empirically derived an index to distinguish biological from chrono- 
logical ageing with a result that seems to offer reasonable precision. For example, equation 2 predicts a maximum life span 126 years, compared with the maximum-recorded life span of about 122 years. This is of interest, but we have to be careful with its interpretation. We are not claiming that 126 years is the absolute limit of the human lifespan. Note that this is an average characteristic of the sample, in which older adults with deficits have been over-represented. Moreover, as has been well argued, there is no evidence for such a limit, and considerable evidence against it [30]. The accumulation of deficits in our equations suggests a process whereby damage is initially compensated by redundancy of systems, but when the redundancy is exhausted (i.e., too many deficits are accumulated) any new insult leads to death [31]. Similarly, equation (1) corresponds to the differential equation relating the proportion of the deficits $q$ to the instantaneous rate of increase in deficits; i.e. $d q / d t: d q / d t=k q(k=0.03)$. In other words, the average annual accumulation of deficits in successful ageing (in this case for individuals with no cognitive impairment) is $3 \%$ per year.

Here we used death as the outcome. However, other adverse outcomes, such as institutionalisation can also be considered and will be the subject of further inquiry. This approach also incorporates an important feature of frailty, which has otherwise received little attention in its operationalization, namely its relationship to age. While ageing is readily accepted as being associated with frailty, as argued elsewhere, $[1,26]$ the notion of frailty finds its roots in the imprecision of chronological age as an explanatory model in predicting adverse outcomes in individual cases. As presented here, $P B A$ represents the individual case, but its relationship to age is inherent in the definition. As noted (Figure 2), individual values of the impairment index can vary widely at any given $C A$. As a statistical analogy, $P B A$ for a subject represents their individual value; $C A$ represents the mean. In other words, by taking into account both the proportion of deficits accumulated by an individual at a given age, and the average proportion of deficits estimated from the successful ageing group, we are able to describe respectively, an individual or a characteristic of a population. While this is of interest, and served as the basis of further inquiry [27] we are not making a claim to have definitively calculated the PBA. Rather, we have presented one method of so doing, and note that this approach has properties which encourage us to pursue the analysis further in other databases.

\section{Conclusions}

A frailty index can be estimated as the proportion of deficits older adults accumulate over time. By knowing the proportion of deficits a given individual has accumulated at a particular age, personal biological age can be estimated. Personal biological age for a given individual can be estimated from their frailty index value, as the age at which the person has accumulated that frailty index value. Personal biological age is a stronger correlate of mortality than chronological age. Relative frailty and fitness can therefore be estimated as the difference between chronological and biological age. The frailty index is a sensitive predictor of survival. As the index includes items not traditionally related to adverse health outcomes, the finding is compatible with a view of frailty as the failure to integrate the complex responses required to maintain function.

\section{Competing interests \\ None declared.}

\section{Acknowledgements}

The data were collected as part of the Canadian Study of Health and Aging, funded by the Seniors Independence Research Program, administered by the National Health Research and Development Program (NHRDP) of Health and Welfare Canada (Project No. 6606-3954-MC(S)). This analysis was supported by grants from the National Health Research Development Program of Health Canada (grant no. 6603-|47|-55) and the Queen Elizabeth II Research Foundation. The data reported in this article were collected as part of the Canadian Study of Health and Aging. The core study was funded by the Seniors' Independence Research Program, through the National Health Research and Development Program (project no. 6606-3954$\mathrm{MC}(\mathrm{S})$ ). Additional funding was provided by Pfizer Canada Incorporated through the Medical Research Council/Pharmaceutical Manufacturers Association of Canada Health Activity Program, the National Health Research and Development Program (project no. 6603-I4I7-302(R)). The study was coordinated through the University of Ottawa and the Division of Aging and Seniors, Health Canada. Additional funds for analysis came from Hoechst Marion Roussel Canada, through a grant administered by the Medical Research Council of Canada (grant MRC PA- I3560). Kenneth Rockwood and Janice Graham are supported by Investigation Awards from The Canadian Institute of Health Research. We are grateful to two anonymous referees for their constructive comments.

\section{References}

I. Rockwood K, Fox RA, Stolee P, Robertson D, Beattie BL: Frailty in elderly people; an evolving concept. CMAJ I994, I 50:489-495

2. Reisberg B, Ferris SH, de-Leon MJ, Crook T: The Global Deterioration Scale for assessment of primary degenerative dementia. Am J of Psych 1982, I39: I I36-I I39

3. Hébert R, Brayne C, Spiegelhalter D: Factors associated with functional decline and improvement in a very elderly community-dwelling population. AMJE I999, I 50:50I-5I0

4. Thomas VS, Rockwood K, McDowell I: Multidimensionality in instrumental and basic activities of daily living. J Clin Epidemiol | 997, 5 |:3|5-32|

5. Seeman TE, Berkman LF, Charpentier PA, Blazer DG, Albert MS, Tinetti ME: Behavioural and psychosocial predictors of physical performance: MacArthur studies of successful aging. J Gerontol A Biol Sci Med Sci 1995, 50:MI77-MI83

6. Campbell AJ, Buchner DM: Unstable disability and the fluctuations of frailty. Age \& Ageing 1997, 26:315-318

7. Van Boxtel MP, Buntinx F, Houx PJ, Metsemakers JF, Knottnerus A, Jolles J: The relation between morbidity and cognitive performance in a normal aging population. J Gerontol $A$ Biol Sci Med Sci 1998, 53:MI47-MI54

8. Finch CE: Variations in senescence and longevity include the possibility of negligible senescence. J. Gerontol. Biol. Sci. I998, 53A:B235-B239

9. Rockwood K, Stadnyk K, MacKnight C, et al: A brief clinical instrument to classify frailty in elderly people. Lancet 1999, 353:205206

10. Graham JE, Mitnitski AB, Mogilner AJ, Rockwood K: The dynamics of cognitive aging: distinguishing functional age and disease from chronological age in a population. Am J Epidumiol 1999 , I 50:1045-1054 
1I. Graham JE, Mitnitski AB, Mogilner AJ, Gauvreau D, Rockwood K: Symptoms and signs in dementia: synergy and antagonism. Dementia 1996, 7:331-335

12. Yates FE, Benton LA: Loss of integration and resiliency with age: a dissipative destruction. In: Handbook of physiology: aging |99559|-610

13. Lipsitz LA, Goldberger AL: Loss of 'complexity' and aging. JAMA 1992, 2671:806-809

14. Berg L, Danziger WL, Storandt M, Coben LA, Gado M, Hughes CP, Knesevich JW, Botwinick J: Predictive features in mild senile dementia of the Alzheimer type. Neurology 1984, 34:563-569

15. Small BJ, Backman L: Cognitive correlates of mortality: evidence from a population-based sample of very old adults. Psychol Aging 1997, 12:309-3 I3

16. Canadian Study of Health and Aging Working Group: The Canadian Study of Health and Aging Study methods and prevalence of dementia. CMAJ 1994, I50:899-9/3

17. Teng EL, Chui HC: The Modified Mini-Mental State (3 MS) Examination. J Clin Psychiatry 1987, 48:314-913

18. Graham JE, Rockwood K, Beattie BL, McDowell I, Eastwood R, Gauthier S: Standardization of the diagnosis of dementia in the Canadian Study of Health and Aging. Neuroepidemiology 1996, I5:246-256

19. Graham JE, Rockwood K, Beattie BL, et al: Prevalence and severity of cognitive impairment with and without dementia. Lancet 1997, 349:1793-1796

20. Canadian Study of Health and Aging. Working group: The incidence of dementia in Canada. Neurology 2000, 55:66-73

21. Graham JE, Mitnitski AB, Mogilner AJ, Gauvreau D, Rockwood K: An algorithmic approach to the differential diagnostics of dementua dementia. Dementia 1996, 7:324-330

22. Mitnitski AB, Graham JE, Mogilner AJ, Rockwood K: Vector diagnostics in dementia derived from Bayes' theorem. Am J Epidemiol |997, | 46:665-67|

23. Mitnitski AB, Graham JE, Mogilner AJ, Rockwood K: The rate of decline in functions in Alzheimer's disease and other dementias. J Geront Med Sci 1999, 54A:M65-M69

24. Fayyad UM, Piatetsky-Shapiro G, Smyth P, Uthurusamy R, eds: Advances in Knowledge Discovery and Data Mining. Menlo Park, CA: AAAI Press 1996

25. Tamayo P, Slonim D: Molecular classification of cancer: class discovery and class prediction by gene expression with selforganizing maps: methods and applications to hematopoietic differentiation. Proc Nat Acad Sci USA 1999, 96:2907-29|2

26. Rockwood K, MacKnight C, Hogan D: Conceptualization and instrumentation of frailty. Drugs Aging 2000, 17:295-302

27. Mitnitski AB, Mogilner AJ, Rockwood K: Accumulation of deficits as a proxy measure of aging. The Scientific World 200 I, I:323-336

28. Brown M, Sinacore DR, Binder EF, Kohrt WM: Physical and performance measures for the identification of mild to moderate frailty. Journal of Gerontology: Medical Sciences 2000, 6:M35-M353

29. Ferraro KF, Wilmoth JM: Measuring morbidity: disease counts, binary variables, and statistical power. Journal of Gerontology: Social Sciences 2000, 3:S173-S189

30. Gavrilov LA, Gavrilova NS: The Biology of Life Span. A Quantitative Approach 1991

31. Gavrilov LA, Gavrilova NS: The reliability theory of aging and longevity. J. Theor. Biol. 200I, 2 I 3:527-545
Publish with BioMed Central and every scientist can read your work free of charge

"BioMedcentral will be the most significant development for disseminating the results of biomedical research in our lifetime." Paul Nurse, Director-General, Imperial Cancer Research Fund

Publish with BMC and your research papers will be:

- available free of charge to the entire biomedical community

- peer reviewed and published immediately upon acceptance

- cited in PubMed and archived on PubMed Central

- yours - you keep the copyright

Submit your manuscript here:

http://www.biomedcentral.com/manuscript/

BiolMedcentral.com ditorial@biomedcentral.com 